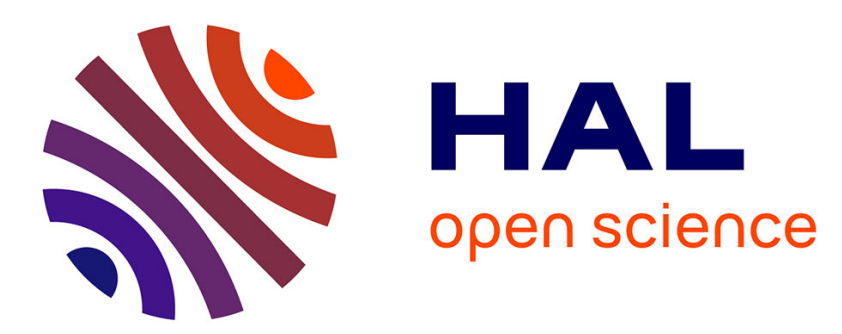

\title{
Identification of the mechanical moduli of flexible thermoplastic thin films using reflected ultrasonic waves: Inverse problem
}

Hacene Lazri, Erick Ogam, Boudour Amar, Z.E.A. Fellah, Andrew O. Oduor, Paul Baki

\section{- To cite this version:}

Hacene Lazri, Erick Ogam, Boudour Amar, Z.E.A. Fellah, Andrew O. Oduor, et al.. Identification of the mechanical moduli of flexible thermoplastic thin films using reflected ultrasonic waves: Inverse problem. Ultrasonics, 2017, 81, pp. 10-22. 10.1016/j.ultras.2017.05.011 . hal-01529180

\section{HAL Id: hal-01529180 \\ https://hal.science/hal-01529180}

Submitted on 30 May 2017

HAL is a multi-disciplinary open access archive for the deposit and dissemination of scientific research documents, whether they are published or not. The documents may come from teaching and research institutions in France or abroad, or from public or private research centers.
L'archive ouverte pluridisciplinaire HAL, est destinée au dépôt et à la diffusion de documents scientifiques de niveau recherche, publiés ou non, émanant des établissements d'enseignement et de recherche français ou étrangers, des laboratoires publics ou privés. 


\title{
Identification of the mechanical moduli of flexible thermoplastic thin films using reflected ultrasonic waves: Inverse problem
}

\author{
Hacene Lazri ${ }^{\mathrm{a}}$, Erick Ogam ${ }^{\mathrm{b}, *}$, Boudour Amar ${ }^{\mathrm{a}}$, Z. E. A Fellah ${ }^{\mathrm{b}}$, Andrew O. Oduor ${ }^{\mathrm{c}}$, \\ Paul Baki ${ }^{\mathrm{d}}$ \\ ${ }^{a}$ Laboratoire d'Elaboration et d'Analyse des Matériaux, Université BADJI Mokhtar Annaba, Algeria \\ ${ }^{b}$ Laboratoire de Mécanique et d'Acoustique, CNRS, UPR 7051, Aix-Marseille Univ, Centrale \\ Marseille, F-13453 Marseille Cedex 13, France \\ ${ }^{c}$ Maseno University, Department of Physics and Material Sciences, Maseno, Kenya \\ ${ }^{d}$ Technical University of Kenya, Faculty of Applied Science and Technology, Haile Selassie Ave, \\ Nairobi, Kenya
}

\begin{abstract}
A method for the identification of the mechanical moduli and density of flexible, supple thermoplastic thin films placed on elastic substrates using ultrasonic waves has been developed. The composite medium immersed in a fluid host medium (water) was excited using a 50Mhz transducer operating at normal incidence in reflection mode. Inverse problems involving experimental data pertaining to elastic wave propagation in the thin films on their substrates and theoretical fluid-solid interaction models for stratified media using elasticity theory were solved. Two configurations having different interface boundary conditions (BC) were modeled, transverse slip for the sliding contact interface in the case where the thin films were placed on the substrate without bonding; a bonded interface condition. The inverse problem for the recovery of the mechanical parameters were solved for the thin films under the bonded and slip BCs. Substrates made of different elastic materials having different geometries were also evaluated and their advantages discussed.
\end{abstract}

Keywords: Thin solid flexible films, substrate, ultrasound, Elasticity, layered media, inverse problem, mechanical moduli,

\footnotetext{
*Principal corresponding author
} 


\section{Introduction}

This paper reports the development of new methods for the characterization of two types of thin flexible films made from thermoplastic polymers by solving an inverse problem using real ultrasound reflection data and theoretical interaction models. The first set of films were thin flexible, semi-crystallin thermoplastic polymer resin films made from polyethylene terephthalate (PET) [1] and polycaprolactone (PCL) triol that are employed in a variety of applications. These are the most common films of the polyester family. PET like the majority of thermoplastics, can be bi-axially oriented or bubble extruded and engineered to meet specific needs. Their mechanical properties make them the premier film choice for a variety of industries. They are some of the most common substrates used in the converting industry because of their balance of properties as compared to other thermoplastic polymers. They are better than other thermoplastics in the following categories: tensile strength, tear strength. They are employed in applications like; packaging films, graphics/print media, custom engineered coated films, insulation films, surface protection films/hardcoats, photo-voltaic back sheets. They are also widely used as protective films that help hold shattered glass windows together during severe weather conditions, accidental impact or vandalism.

The second type of thin film characterized in this study is a dominant interlayer material made from polyvinyl butyral (PVB). It is prepared from polyvinyl alcohol by reaction with butyraldehyde. PVB thin films are soft and flexible and are often employed as resin for applications that require strong binding, optical clarity, adhesion to many surfaces, toughness and flexibility. The major application of PVB is laminated safety glass for automobile windshields. The PVB interlayer functions to distribute impact forces across a greater area of the glass panes, thus increasing the impact resistance of the glass [2]. PVB is also used in photo-voltaic thin film solar modules [3].

The desire to improve these materials and their manufacturing processes to obtain excellent properties requires that methods of recovery of their mechanical properties be developed. The manufacturing processes can generate a few challenging characteristics 
like: variability, non-uniformity, and instability of attributes that can only be unmasked if precise characterization methods exist. Knowledge of elastic properties is also important in estimating residual stress, to correlate with their chemical structure [4] for verifying the micro-structure, to assess adhesion and provide better predictions of the laminated glass window response modeling [5], for facilitating analysis of other characteristic modifying the film parameters e.g atmospheric pressure plasma parameters after the PET has been treated with helium plasma [6].

The popular methods for the characterization of thin films are based on scanning acoustic microscopy [7]. These methods have mainly allowed access to the measured velocity of sound waves propagating in the thin films and their output voltage $V(z)$ of the focused transducer curves as a function of the distance $(z)$ of the focus and surface of the specimen [8] at a frequency range from 170-450 MHz. Lavrentyev et al [9] proposed a method for determining the properties of a coating on a thin plate. The method allows simultaneous determination of the coating thickness, density, elastic moduli and attenuation from both the normal and oblique incidences reflection frequency spectra. Phase-shift shadow Moiré optical methods have also been employed to recover Young's moduli of composite systems composed of various epoxy coated polyethylene terephthalate (PET) micro-cantilevers [10].

In this paper a model comprising of equations for the acoustic wave propagation in the host medium and the elastic wave propagation in the layered composite medium composed of the thin films and their substrates for the geometrical configurations studied have been developed using elasticity theory [11]. The detailed theory of wave reflection from interfaces often derived using acoustic impedance theory in layered media are discussed in detail in [12]. In this study only the mechanical properties of the thin films were recovered. 


\section{Experimental methods and configurations}

Thin thermoplastic films are flexible, supple materials therefore in order to be able to characterize them using plane acoustic waves requires that they either be placed to rest on or glued onto an elastic substrate. In this study the substrates employed were made from different elastic materials in form of thick plates, cylinders and slabs. The experiments were done at normal incidence using the pulse-echo technique in which a short, broad-band pulse (E) is sent by a transmitting transducer. The pulse reflected (R) back by the front surface of the immersed film on a substrate were received by the same transmitting transducer (Fig. 10). In order to consider the substrate layer as a semi-infinite half space, the signals reflected from its opposite face were not included in the captured transducer signals' windows. 

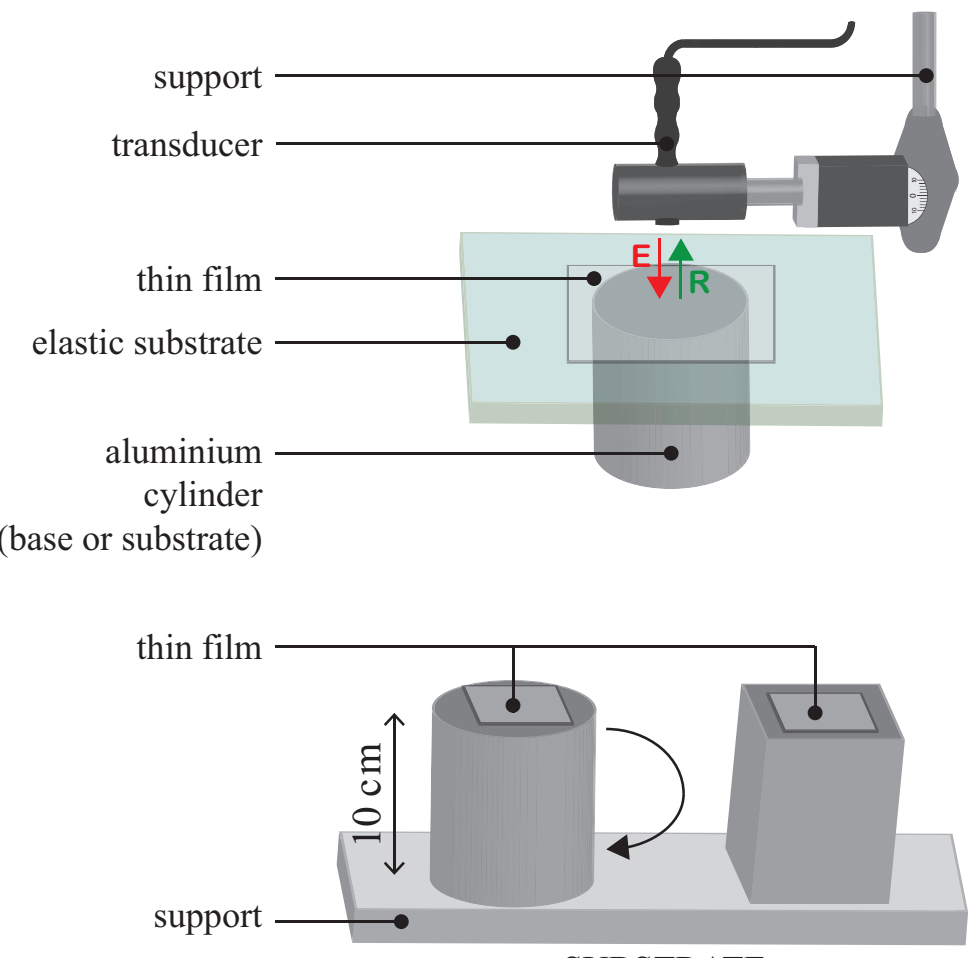

SUBSTRATE

Figure 1: The experimental setup for the characterization of thin films using reflected ultrasonic waves. The upper panel depicts the the film glued onto an elastic plate acting as substrate. The two aluminium substrates of different geometries are also shown. The difference between the two lower substrates is that the cube has a rougher surface.

The thin films and their substrates were placed on an elastic cylinder acting as support. This was done in this configuration to elevate the height of the thin film on its substrate so as to approach the transducer as shown on the upper panel of Fig. (11). In other configurations, the elastic cylinders of circular and square cross-sections were employed as substrates. In order to acquire the incident signal, the film-substrate pair were turned upside down so that the lower side of the substrate acted as an acoustic mirror (lower panel of Fig. (1)). In that position the substrate was resting on the film. This was done to assure that the reflector-transducer distance were approximately the same as the film-transducer one when acquiring the reflected incident signal.

The high frequency immersion transducer employed in this study was a $50 \mathrm{MHz}$ 
V358/50/0.25" connected to a pulser Model 5077PR both manufactured by Panametrics (Waltham USA). The signals were digitized (after averaging of 512 samples) using a Tektronix oscilloscope model TDS3014B (Beaverton, OR USA). The instrumental setup and acquisition methods are exposed in details in reference [13].

\subsection{Matching the transducer-reflector and transducer-film surface distances}

As mentioned previously, the reflected incident signal was obtained by turning the film-substrate pair upside down. In theory this assured that the transducer-reflector and transducer-film surface distances were the same when acquiring the reflected incident signal and the one reflected by the film. However, the thin films being flexible and supple, the weight of the substrate squeezed them resulting in the two distances not being equal. Consequently the arrival times of the incident and the reflected signals were different. This was later corrected artificially so that the arrival times of the two signals were both the same on the time axis. This was done practically by shifting the signals so that the reflected wave at the first interface of the film was at the same position temporal-wise with that from the reflector. This was subject to two conditions: The two signals should not be deformed (like in the case of non-normal incidence $\left(\theta_{i} \neq 0\right)$, nor distorted by the transducer signal amplifier due to high amplification levels). Distortion also occurs if the two angles of incidences are not the same between the two measurements to acquire the reflected and the incident waves. The best indication is that the two temporal signals should be of Gaussian form after being Fourier transformed. This correction also assured that the magnitude of the reflection coefficient was correct.

\section{Modeling the configurations}

The geometry of the acoustic wave reflection configuration by the layered media composed of the thin film $\left(\Omega_{1}\right)$ placed on an elastic substrate $\left(\Omega_{2}\right)$ and immersed in water $\left(\Omega_{0}\right)$ are depicted in Fig. (2). The mode conversions are also represented in the figure. The geometry is represented with an oblique angle of incidence $\left(\theta_{i} \neq 0\right)$ 
for the acoustic wave impinging on the film. The measurements were done at normal incidence $\left(\theta_{i}=0\right)$ only. The theoretical models herein were developed using elasticity theory (starting from Hooke's law up to the derivation of the elastic wave propagation equations in the elastic solid as in reference [11]). The general case of an oblique angle of incidence $\theta_{i}$ was chosen for the model.

The plane harmonic wave solutions of the wave equations (in the Laplace domain $s=\mathrm{i} \omega)(\mathrm{i}=\sqrt{-1}$ and $\omega$ is the angular frequency) in terms of potentials (compressional $\Psi$ and shear $\Phi)$ for each domain are

$$
\begin{array}{r}
\Psi^{s 1}(x, z, s)=\left(A_{11} \cosh \left(\kappa z_{11} z\right)+A_{12} \sinh \left(\kappa z_{11} z\right)\right) \mathrm{e}^{\kappa_{f} x} z \in \Omega_{1}, \\
\Phi^{s 1}(x, z, s)=\left(B_{11} \cosh \left(\kappa z_{31} z\right)+B_{12} \sinh \left(\kappa z_{31} z\right)\right) e^{\kappa_{f} x} z \in \Omega_{1}, \\
\Psi^{s 2}(x, z, s)=\Phi_{21} \mathrm{e}^{\kappa z_{12}(z+L)} \mathrm{e}^{\kappa_{f} x} z \in \Omega_{2}, \\
\Phi^{s 2}(x, z, s)=\Psi_{21} \mathrm{e}^{\kappa z_{32}(z+L)} \mathrm{e}^{\kappa_{f} x} z \in \Omega_{2}, \\
p_{f}(x, z, s)=\left(\mathrm{e}^{\kappa_{f} \cos (\theta) z}+R^{I M} \mathrm{e}^{-\kappa_{f} \cos (\theta) z}\right) \mathrm{e}^{\kappa_{f} x} p_{i} \quad z \in \Omega_{f}\left(\Omega_{0}\right),
\end{array}
$$

where the superscripts $s$ and $f$ stand for solid and fluid media respectively, $p_{i}$ is the amplitude of the incident compressional wave in the fluid, $R^{I M}$ is the reflection coefficient, the compressional wave potential amplitudes $A_{11}=\Phi_{11}+\Phi_{12}, A_{12}=\Phi_{11}-\Phi_{12}($ when $\Psi^{s 1}(x, z)$ is written in exponential forme and $A_{11}$ and $A_{12}$ are the amplitudes), and those of the shear waves are $B_{11}=\Psi_{11}+\Psi_{12}$ and $B_{12}=\Psi_{11}-\Psi_{12}$. The amplitude $\Phi_{11}$ and $\Psi_{11}$ are for the incident P-wave and shear-wave respectively, while $\Phi_{12}$ and $\Psi_{12}$ are amplitude of the reflected $\mathrm{P}$-wave and shear-wave respectively and $L$ is the thickness of the film.

The complex wave-numbers $\kappa_{f}=\mathrm{i} \gamma_{f}, \gamma_{f}=k_{f} \sin (\theta)\left(k_{f}\right.$ is the compressional wave number in the fluid) and the complex wave numbers $\kappa z_{1 n}$ and the shear ones $\kappa z_{3 n}$ 
$\left(n=1,2\right.$ represents the medium $\left.\Omega_{n}\right)$ are given by

$$
\begin{aligned}
& \kappa z_{11}=\mathrm{i} \sqrt{k_{11}^{2}-\gamma_{f}^{2}}, \quad \kappa z_{12}=\mathrm{i} \sqrt{k_{12}^{2}-\gamma_{f}^{2}} \\
& \kappa z_{31}=\mathrm{i} \sqrt{k_{31}^{2}-\gamma_{f}^{2}}, \quad \kappa z_{32}=\mathrm{i} \sqrt{k_{32}^{2}-\gamma_{f}^{2}}
\end{aligned}
$$

In terms of the elastic constants, the wave numbers are given by

$$
\begin{aligned}
& k_{11}=\omega \sqrt{\frac{\rho_{1}}{M_{1}}}, \quad k_{31}=\omega \sqrt{\frac{\rho_{1}}{\mu_{1}}} \\
& k_{12}=\omega \sqrt{\frac{\rho_{2}}{M_{2}}}, \quad k_{32}=\omega \sqrt{\frac{\rho_{2}}{\mu_{2}}}
\end{aligned}
$$

where $\rho_{n}, \mu_{n}$ and $M_{n}(n=1,2)$ are the density, shear modulus and the P-wave, modulus of layer $n$ respectively. The inherent damping of the the films were taken into account by introducing a scalar-valued loss factor $\eta_{n}^{s}$ into Young's modulus, such that $E_{n}^{s}=E_{n}^{s}\left(1+\mathrm{i} \eta_{n}^{s}\right)\left(E_{n}^{s}\right.$ is the dynamic Young's modulus of the layer). The relationships between $M_{n}$ and $E_{n}^{s}$ are given in [11]. 


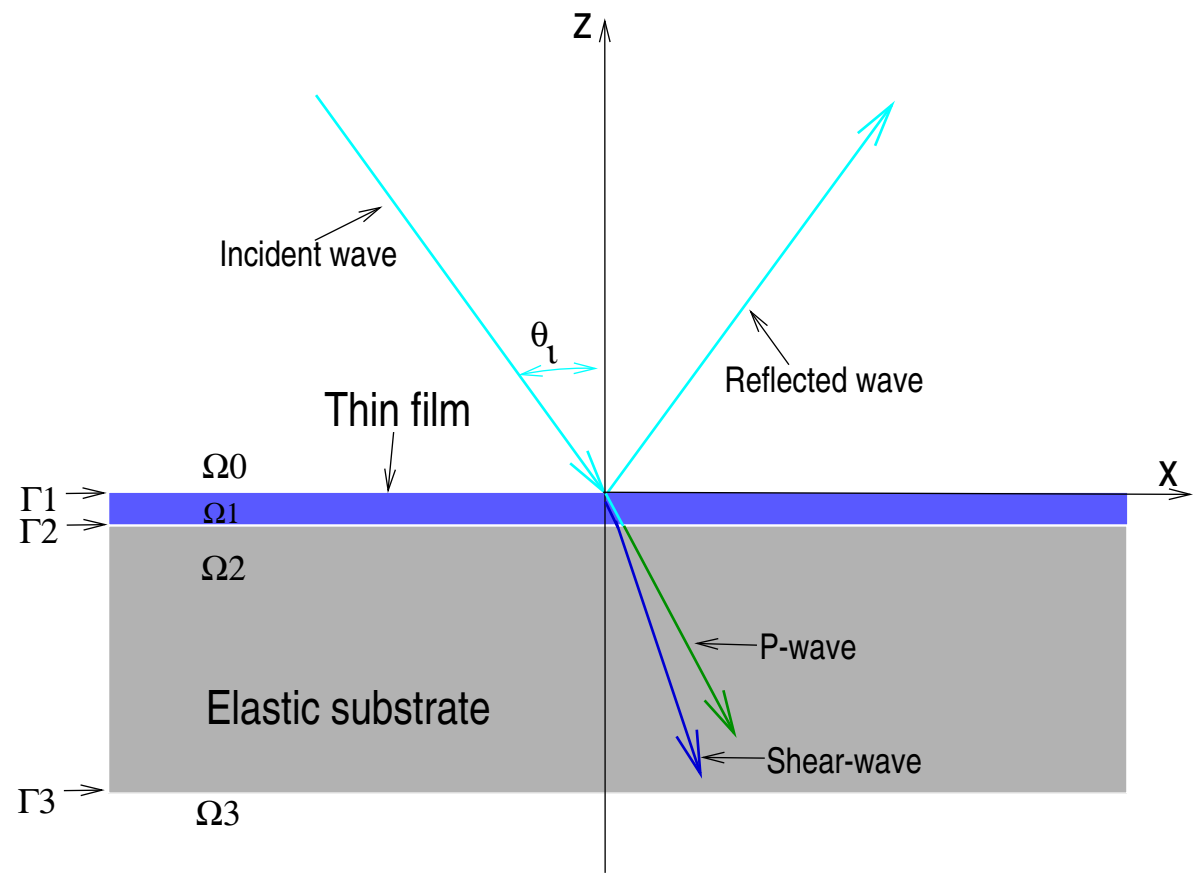

Figure 2: The geometry of the reflection configuration of ultrasonic waves impinging on a thin film (domain $\Omega_{1}$ ) placed on an elastic substrate $\left(\Omega_{2}\right)$. The semi-infinite fluid media (water) domain is depicted by $\Omega_{0}$. The boundary between the fluid and the film is $\Gamma_{1}$, that with the substrate $\Gamma_{2}$ and that between the substrate and the fluid $\Omega_{3}$, is $\Gamma_{3}$. The mode conversions of the incident wave from P-wave into P-wave and shear waves in the thin film $\left(\Omega_{1}\right)$ are not shown but are indicated for the substrate.

The probing frequency is imposed by the film intrinsic properties (density, elastic moduli), thickness $(L)$ and the number of desired longitudinal modes $(n)$ propagating in the film. The longitudinal frequency modes can be determined using,

$$
f_{n} \approx n v_{11} / 2 L,
$$

where $v_{11}=k_{11} / \omega$ is the longitudinal wave velocity (Eqn. 3). The probing frequency within the resonant region is important for a good signal-to-noise ratio. The reduction in film thickness raises the required probing ultrasonic frequency even higher. 


\subsection{Thin flexible film stuck on an elastic substrate}

In this configuration the thin film $\left(\Omega_{1}\right)$ was glued onto the elastic substrate of finite thickness. To simplify the modeling, the elastic substrate was considered as a semiinfinite elastic half-space at the wavelengths employed i.e no reflected wave from the second interface $\Gamma_{3}$ of the elastic medium substrate (top panel of Fig⿴). The geometry of the problem is depicted in Fig. (2). The upper surface $\left(\Omega_{0}\right)$ is considered as a semiinfinite fluid (water) half-space.

The boundary conditions at the interface $\Gamma_{1}$ between the thin film and the upper fluid are ; continuity of the velocity and the normal stress $\left(\sigma_{z z}\right)$, while the tangential stress $\left(\sigma_{x z}\right)$ is zero across the interface at $z \in \Gamma_{1}$

$$
v^{f}(x, 0, s)=v_{z}^{s 1}(x, 0, s), \quad p_{f}(x, 0, s)=\sigma_{z z}^{s 1}(x, 0, s), \quad \sigma_{x z}^{s 1}(x, 0, s)=0 .
$$

The boundary conditions at the interface $\Gamma_{2}$ between the thin film and the elastic substrate are bonded together (glued condition) are; continuity of the velocity, the normal stress and tangential stress across the interface at $z \in \Gamma_{2}$,

$$
\begin{aligned}
& v_{z}^{s 1}(x,-L, s)=v_{z}^{s 2}(x,-L, s), \quad \sigma_{z z}^{s 1}(x,-L, s)=\sigma_{z z}^{s 2}(x,-L, s), \\
& \sigma_{x z}^{s 1}(x,-L, s)=\sigma_{x z}^{s 2}(x,-L, s) .
\end{aligned}
$$

The displacement, velocity, stress and strain relationships are given in AppendixA and the resulting matrix equation obtained using the elasticity relations is given in AppendixA.1. It is a linear system of the form $\mathbf{A} \cdot \mathbf{x}=\mathbf{b}$ and was solved using a linear solver that returns vector $\mathrm{x}$ that satisfies the equation[14]. The reflection coefficient $R^{I M}$ was then extracted from the solution of this equation. This constitutes the interaction model (IM). 


\subsection{Thin flexible films resting on a substrate of finite thickness}

The thin film is bounded on one side by a semi-infinite fluid half-space $\left(\Omega_{0}\right)$ while the opposite side is bounded by a substrate considered as a semi-infinite elastic half space.

The boundary conditions at the interface $\Gamma_{1}$ between the thin film and the fluid are: continuity of the velocity and the normal stress while the tangential stress $\left(\sigma_{x z}\right)$ is zero across the interface at $z \in \Gamma_{1}$,

$$
v^{f}(x, 0, s)=v_{z}^{s 1}(x, 0, s), \quad p_{f}(x, 0, s)=\sigma_{z z}^{s 1}(x, 0, s), \quad \sigma_{x z}^{s 1}(x, 0, s)=0 .
$$

The thin film was placed on the substrate and the water between them was squeezed out by sliding the edge of a flat ruler over it. The measurement was taken immediately before water infiltrated between the film and substrate interface. The boundary conditions between the thin film and the elastic substrate at the interface $\Gamma_{2}$ was considered to be that of contact allowing transverse slip (sliding can occur). Overall, there was; continuity of the normal velocity, the normal stress, while the tangential stress $\left(\sigma_{x z}\right)$ is zero across the interface at $z \in \Gamma_{2}$,

$$
\begin{gathered}
v_{z}^{s 1}(x,-L, s)=v_{z}^{\mathrm{s} 2}(x,-L, s), \quad \sigma_{z z}^{s 1}(x, z, s)=\sigma_{z z}^{s 2}(x,-L, s), \\
\sigma_{x z}^{s 1}(x,-L, s)=\sigma_{x z}^{s 2}(x,-L, s)=0 .
\end{gathered}
$$

The resulting matrix equations are given in AppendixA.2. The linear matrix system of equations is solved as in the glued/bonded case in the previous section.

\section{The inverse problem for the retrieval of mechanical parameters of the thin film}

The first inverse problem solved in this study was that of the retrieval of the elastic moduli (Young's modulus, Poisson ratio) and the density parameters from the measured reflection coefficient of the thin film glued or placed on an elastic substrate and both 
immersed in a water tank. The viscoelastic interaction model (IM) of the wave reflection of the setup geometry was developed in this study. An error functional expressing the distance between the reflection coefficient experimental data from that of the interaction model was computed in the interval of the observation frequency, for each set of trial values of the parameters.

$$
\mathfrak{J}\left(E_{1}, \nu_{1}, \rho_{1}, E_{2}, \nu_{2}, \rho_{2}\right)=\sum_{n=1}^{N_{s}}\left(\left|R^{I M}\left(\omega_{n}, E_{1}, \nu_{1}, \rho_{1}, E_{2}, \nu_{2}, \rho_{2}\right)\right|-\left|R^{\exp }\left(\omega_{n}\right)\right|\right)^{2},
$$

where $R^{\exp }$ is the reflection coefficient of the thin film on its substrate, captured from the water tank experimental data and $R^{I M}$ is the interaction model reflection coefficient and $N_{s}$ is the number of samples representing the interval of the observation frequency. The method of signal processing for obtaining the reflection coefficient from the measured incident and reflected signals from the thin film by computing the transfer function, are explained in detail in reference [15]. The Poisson ratios, Young's moduli and densities of the layers are denoted by $\nu_{m}, E_{m}, \rho_{m}(m=1,2)$, respectively. The parameters were retrieved by minimizing the objective functional $\mathfrak{J}$. The substrate parameters $E_{2}, \nu_{2}, \rho_{2}$ were supposed known.

The problem was split into the computation of three cost functions $\left(\mathfrak{J}_{E}, \mathfrak{J}_{\nu}, \mathfrak{J}_{\rho}\right)$ where each of the model parameters $\left(E_{1 s_{m}}, \nu_{1 s_{m}}, \rho_{1 s_{m}}\right.$, where $s$ is the step, $m=1 \ldots p$ and $p$ is the number of intervals) was varied while the other two were fixed. This was done in various intervals not too wide, e.g. Poisson ratio interval was chosen from 0.1 to 0.5 , density from 800 to 2000 , etc.

Three initial values of the sought-for film parameters $\left(E_{1 s i}, \nu_{1 s i}, \rho_{1 s i}\right)$ were chosen at the initial step. Some of these values, found in the literature (e.g for PET [16, 17], for both PET and PVB [18]) were obtained using different methods. The solution for each of the parameters was found from the position of the global minimum of the cost function $(\mathrm{CF})$ exhibiting a parabolic shape. The values corresponding to the three CF minima consti- 
tuted the solution to the inverse problem for the step. The three new minima values were then used to update the initial values for the next step. The search for the optimized values was a manual iterative process repeated for each parameter at a time, at each iteration/step, until convergence was achieved for all the three sought for parameters.

The objective functional curves for each parameter were then reported after assuring that all the three parameters had converged to their optimal values. The curves were plotted with respect to one of the three problem variables, the others being fixed at their computationally-optimal values, i.e, as 2-D plots [19].

The presence of several local minima in the cost functionals can complicate the choice of the correct parameter values [19] but in this study the measured wave velocity and density allowed an unambiguous determination of the parameters. The densities of the films were determined from their precisely measured weights (using Lab Scale Analytical Balance, Sartorius ME235P, Goettingen Germany) and volumes (using a $250 \mathrm{ml}$ graduated cylinder, tolerance $\pm 1.5 \mathrm{ml}$, Witeg, Germany). The two were then compared to the recovered longitudinal wave velocity $v_{11}=\sqrt{M_{1 s i} / \rho_{1 s i}}$ (Eqn. 3) and the film density $\rho_{1 s i}$.

\subsection{Sensitivity of the interaction model to the desired input variation and search for optimized values}

A sensitivity study of the model output to the variation of the desired input to be recovered by the inverse problem (IP) was necessary. This was undertaken prior to solving the IP. It was done to assure that the interaction model was sensitive to the variation of each of the three input variables, a condition that was necessary for the successful recovery of the desired parameters from the experimental data. 


\section{Results}

\subsection{Characterization of thin films glued onto a thick elastic substrate}

\subsubsection{Correction for the time of flight for the incident and reflected signals and the wave}

velocity in the film layer

A correction was undertaken by shifting the signals so as to assure artificially that the transducer-film and transducer-reflector distances were the same. The opposite face of the substrate was used as mirror to obtain the incident signal. Aligning the signals was done by first computing an unbiased estimate of the cross-correlation [20] between the two discrete-time sequences of the acquired signals $\left(p_{i}\left(t_{n}\right), p_{r}\left(t_{n}\right), t_{n}(n=1,2 \ldots)\right.$ are the sequences). The lag $\left(\operatorname{lag}_{1}\right)$ in number of samples was obtained from the maximum of the cross-correlation function between the two measurements. The pulse that correlated best with the incident pressure was found to be the acoustic pressure pulse in the reflected signal corresponding to the reflection from the film-substrate interface $\Gamma_{2}$. The correlation coefficient for the pulse in the reflected signal due to the presence of the fluid-film interface $\Gamma_{1}$, was found to be lower in all the cases.

The best correlated pulse signal peaks from the two measurements were then aligned through shifting of $p_{i}\left(t_{n}\right)$ to obtain $p_{i}^{\prime}\left(t_{n}\right)$ and the tail of $p_{r}\left(t_{n}\right)$ was cut appropriately to obtain $p_{r}^{\prime}\left(t_{n}\right)$ so that the two signals were of same lengths (Fig. 33-2).

The final shift operated assured that the incident signal pulse (in $\left.p_{i}^{\prime}\left(t_{n}\right)\right)$ was aligned with that from the film-water interface $\Gamma_{1}$ in $p_{r}^{\prime}\left(t_{n}\right)$. This was done in two steps. Firstly a division of each element of the new array $p_{r}^{\prime}\left(t_{n}\right)$ with the corresponding element of $p_{i}^{\prime}\left(t_{n}\right)$ $\left(p_{r i}(t)=p_{r}^{\prime}\left(t_{n}\right) / p_{i}^{\prime}\left(t_{n}\right)\right.$ provided a means of destroying the correlation at the points where the pulses of the two signals were already aligned $\left(p_{i}^{\prime}\left(t_{n}\right)\right.$ (Fig. 3 $\left.3-3\right)$. However, the division by $p_{i}^{\prime}\left(t_{n}\right)$ was avoided incase some of its values were null. Instead, the values around the peak of interest were simply annulled. A correlation operation between $p_{i}^{\prime}\left(t_{n}\right)$ and $p_{r i}(t)$ was computed to get the new lag $\operatorname{lag}_{2}$ to be used to shift the signal to the position corresponding to the reflection from the film-water interface $\Gamma_{1}$ (Fig. [3b). This lag constitutes the time lapse for the wave to travel forth and back within the film layer. 
This method was found to be precise and was automated to determine the wave velocities within the film layers. The longitudinal wave velocity was computed using the relation, $v_{L}=2 L /\left(\operatorname{lag}_{2} / F_{s}\right)$ (where $F_{s}$ is the sampling frequency). The frequency spectrum of the different signals are shown in Fig (3)c. The reflection coefficient was computed using the Matlab [21] function tftestimate with a periodic rectangular windowing function [22] applied to section the incident and reflected (shifted and aligned) signals. The reflection coefficients are depicted in (Fig. 3 $\mathrm{d}$ ). When the window function is employed it is not necessary to shift the signals in order to obtain the correct modulus of the reflection coefficient

It can be noted that the first two modes of the film as indicated by the reflection coefficient, are not deformed nor attenuated. The higher modes that occur later on, i.e. after multiple reflections in the film layer are deformed because of interference from reflections originating from the bottom surface of the substrate, $\Gamma_{3}$. In reality, the substrate is not a semi-infinite half-space but an elastic layer having finite thickness. This provides the justification of using a thicker substrate in order to capture many modes of the film before interference from waves propagating within the substrate layer. 

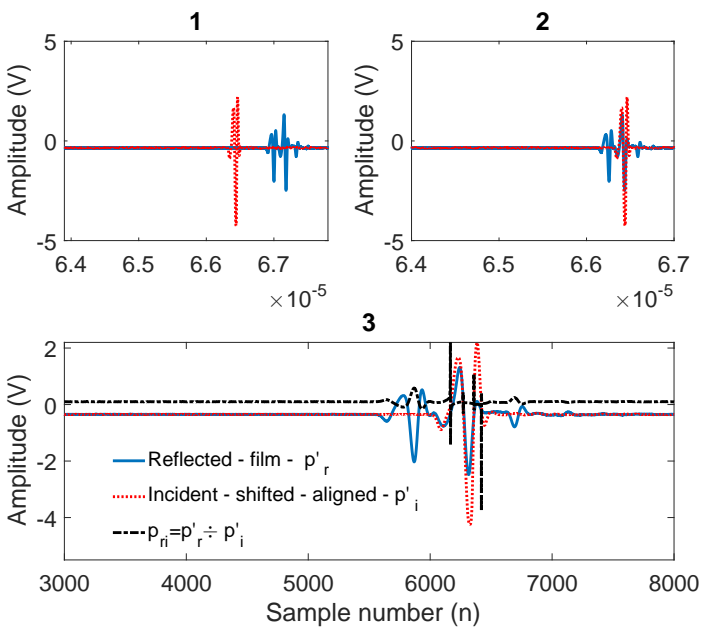

(a)

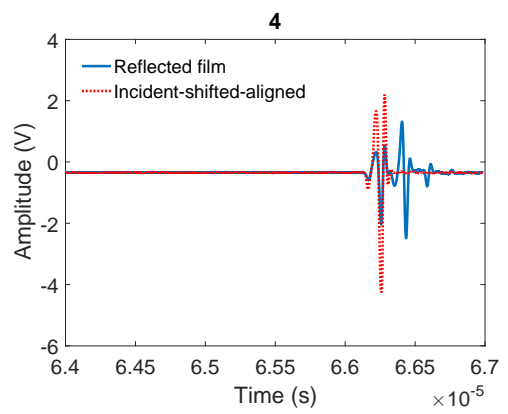

(b)

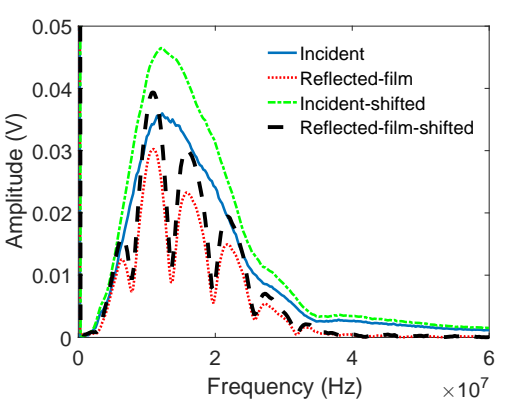

(c)

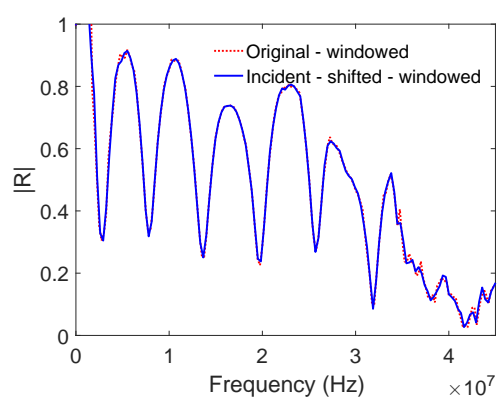

(d)

Figure 3: The corrections done on the temporal signals to make the transducer-film and transducerreflector distances identical (a) Plot number (1), The original temporal incident (using a $1.0 \mathrm{~cm}$ thick aluminum substrate reflector) and the reflected from a $200 \mu \mathrm{m}$ PCL triol film waves (upper left panel numbered (1)). In (2), the shifted incident pulse is aligned with the one reflected from the film-substrate interface $\Gamma_{2}$. In (3), the shifted incident pulse is aligned with the pulse reflected at the interface $\Gamma_{1}$ (b) The shifted and aligned final positions of the two signals. (c) The spectrum of the original and the shifted and aligned signals. They have a Gaussian shape. (d) The reflection coefficient of the original signal and that of the shifted and aligned one. 


\subsection{Sensitivity of the interaction model to the desired input variation}

The reflection coefficient curves were plotted (Fig. 4) for two of the three parameters (Young's modulus and Poisson ratio) and for each, three values were chosen: the correct value and the two others $\pm 10 \%$ of it ( $-5 \%$ and $+10 \%$ for Poisson ratio). The computational results showed that the interaction model was sensitive to the variation of each of the three input variables, a condition that is necessary for the successful recovery of the desired parameters from experimental data.

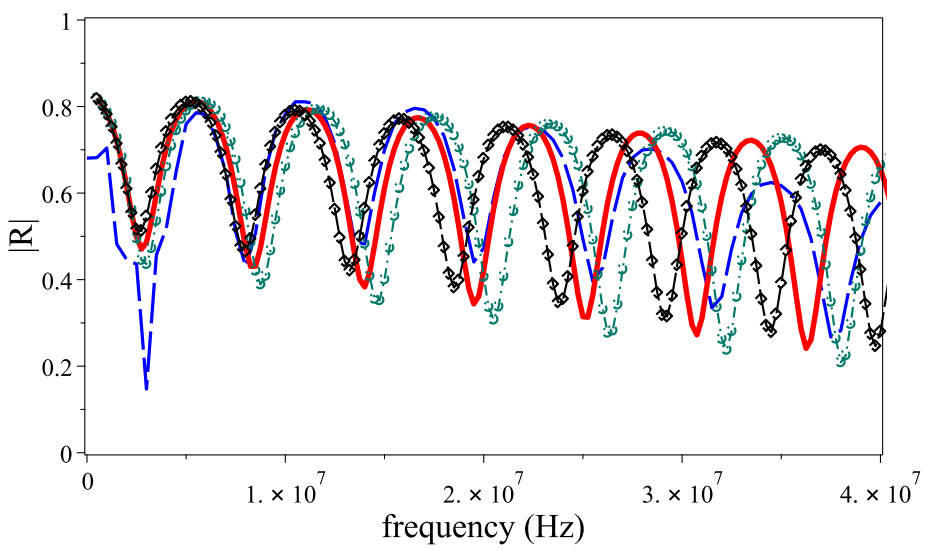

(a)

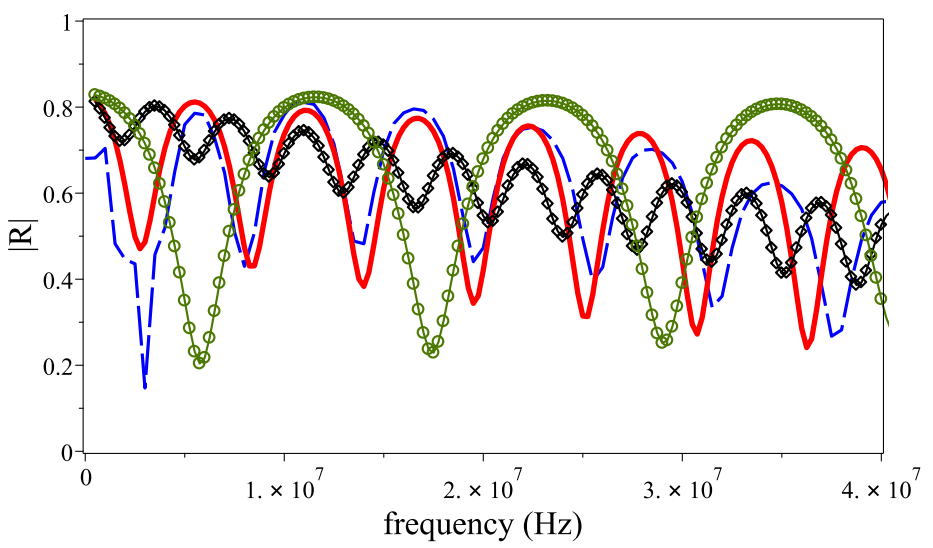

(b)

Figure 4: A sensitivity analysis of the output of the interaction model through variation of the thin film parameters. The theoretical curve (continuous line) using optimized values to fit the experimental data (long-dash line) (a) Variation of Young's modulus, $+10 \%$ (circular marker), $-100 \%$ (diamond marker) of the optimal value. (b) Variation of Poisson ratio, $+5 \%$ (circular marker), $-10 \%$ (diamond marker) for a $200 \mu \mathrm{m}$ PCL triol film. 
5.3. The inverse problem for the recovery of the mechanical parameters - the bonded case

The cost functions obtained from the reflection coefficient of a $200 \mu \mathrm{m}$ polycaprolactone triol film stuck on an aluminium slab (2 cm thick ) are depicted in Fig. (5 $\mathrm{k}-\mathrm{c})$. Fig. (15d and 55) shows the comparisons between the amplitudes and arguments of the reconstructed reflection coefficient, using recovered values, and the experimental ones. 


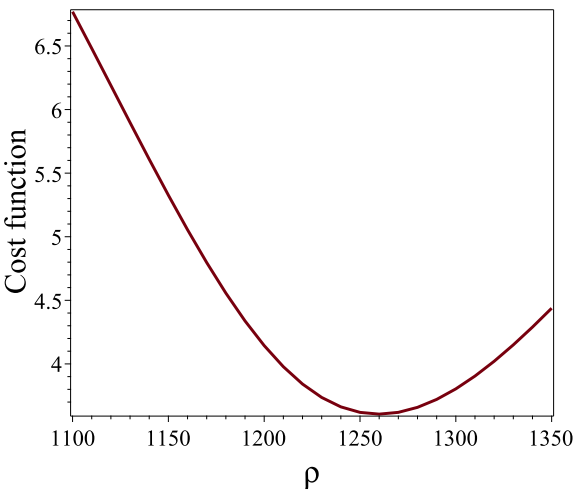

(a)

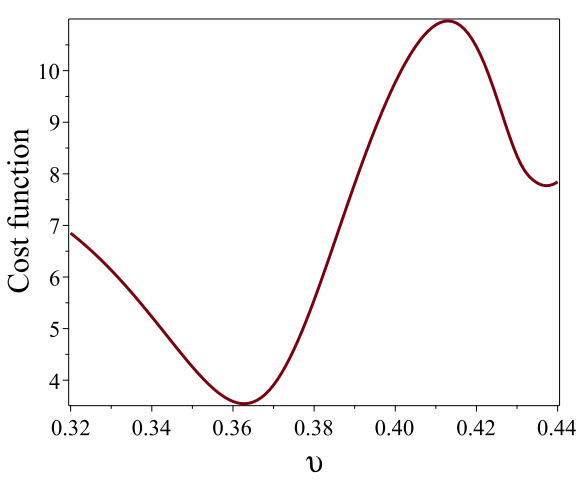

(c)

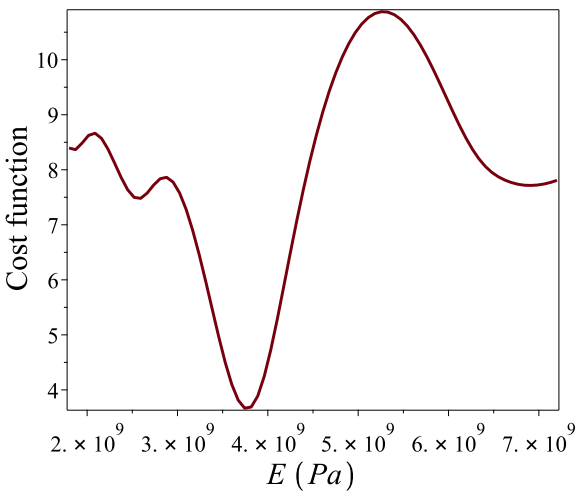

(b)

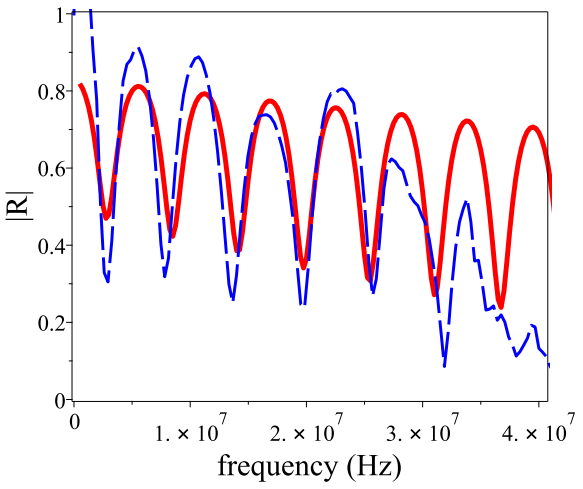

(d)

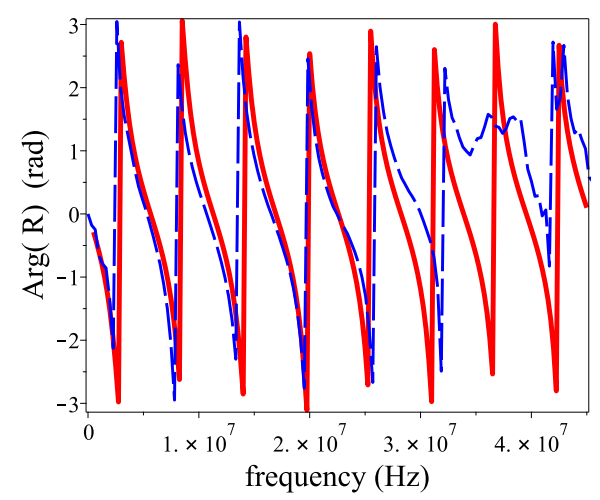

(e)

Figure 5: The cost functions for the recovery of the mechanical parameters for a $200 \mu \mathrm{m}$ polycaprolactone triol film (a) density $\left(\mathrm{kg} / \mathrm{m}^{3}\right)$, the measured one is $1241 \mathrm{~kg} / \mathrm{m}^{3}$, (b) Young's modulus, (c) Poisson ratio. The comparison between the theoretical (continuous line) reflection coefficient using the recovered parameters and the experimental one (long-dash line) (d) amplitude (e) argument. 
The cost functions obtained from the reflection coefficient of a $100 \mu \mathrm{m}$ PET glued on an aluminium cylinder substrate are depicted in Fig. (6). The amplitudes and arguments of the reconstructed reflection coefficient and the measured ones for the aluminium cylinder substrate are also shown. The one for the $8 \mathrm{~mm}$ thick glass plate substrate are also shown. The reflection coefficient for the first longitudinal mode is higher because the amplitude of the incident signal reflected from the glass is slightly smaller than that from the aluminium substrate (employed as mirror/reflectors). 


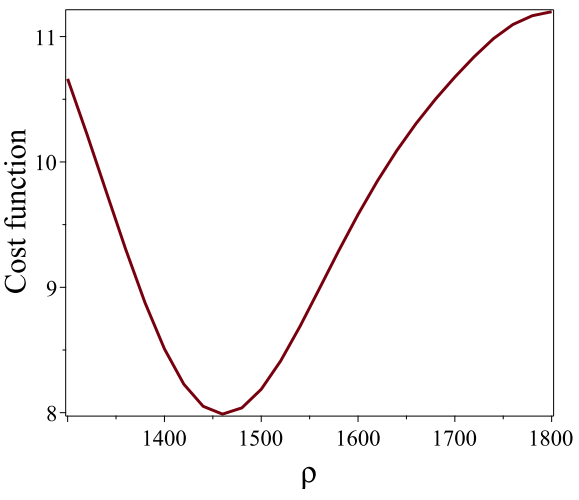

(a)

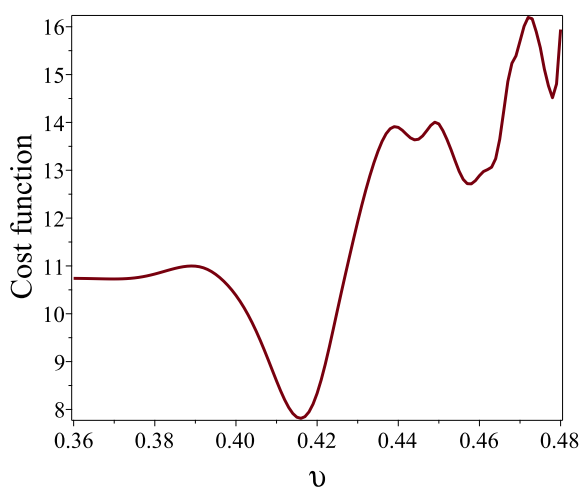

(c)

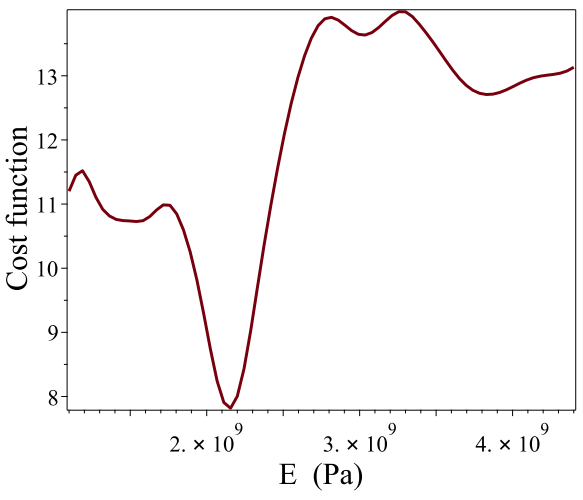

(b)

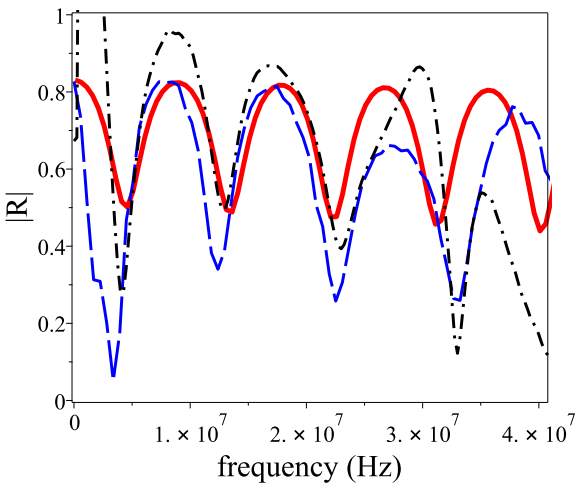

(d)

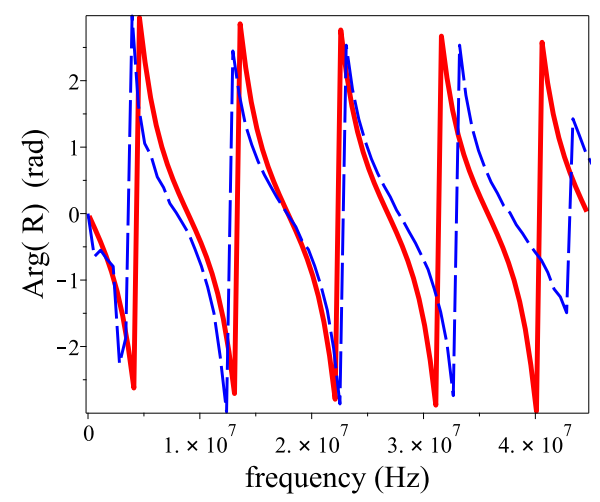

(e)

Figure 6: The cost functions for the the recovery of the mechanical parameters for a $100 \mu \mathrm{m}$ PET film (a) the density $\rho\left(\mathrm{kg} / \mathrm{m}^{3}\right)$, (b) Young's modulus, (c) Poisson ratio. The comparison between the theoretical (continuous line) reflection coefficient using the recovered parameters and the experimental one (cylinder substrate - long-dash and $8 \mathrm{~mm}$ thick glass substrate - dot-dash) (d) the Amplitude (e) the argument. 
It is important that the substrate be of adequate area and thickness. It should be chosen in such a way so as to avoid interference between the waves reflected at the bottom or edges of the elastic substrate with the multiply reflected waves within the film (longitudinal and shear wave modes). The measured reflection coefficient in Fig [6] shows that the higher modes of the longitudinal waves in the film starting from about $25 \mathrm{MHz}$ deviate from the curves provided by the theoretical model. This is due to interference from waves reflected from the lower bottom of the substrate. The thicker the substrate, the later in time the interference with the multiply reflected waves within the thin film. This guaranties the supposed semi infinite half-space dimension condition of the substrate. Since the time window was limited, the oscilloscope time-base was tuned such that these interfering waves were not captured/limited. The first three modes represented by the first three peaks in the reflection coefficient spectrum are enough to obtain the mechanical parameters of the films.

\subsection{The flexible thin PVB films placed on a glass substrate - slip boundary}

The flexible PVB film was placed on a thick glass substrate ( $8 \mathrm{~mm}$ thick) and the reflected signal measured. The substrate was then put under the glass, but supported by the aluminium cylinder acting as platform. Flipping of the thin flexible film-substrate setup upside down was to ensure that the transducer - reflector distance for the reflected incident signal was the same as that of the PVB film - transducer one. The captured reflected wave from a PVB film and the incident wave reflected from flipped substrate face acting as mirror are shown in Fig. (77). The first peaks of the captured temporal incident and reflected pressure signals are not exactly at the same temporal position. This is due to the deformation (squeezing) of the thin flexible PVB film due to the weight of the substrate on the film..

\subsubsection{The inverse problem for the PVB films}

The interface between the thin flexible PVB films and the thick glass plate substrate are considered as having a sliding boundary conditions (BC). Transverse slip is allowed. 
In the interaction model the thick glass substrate is considered as a semi infinite halfspace. The cost functions for the inverse problem of retrieval of the the three sought parameters and the final reflection coefficient reconstructed from these values for the 450 $\mu \mathrm{m}$ thick PVB film are shown in Fig. (8). Two reconstructed reflection coefficients using recovered values for two other thin flexible PVB films of thicknesses $750 \mu \mathrm{m}$ and $400 \mu \mathrm{m}$ are shown in Fig. (9). 

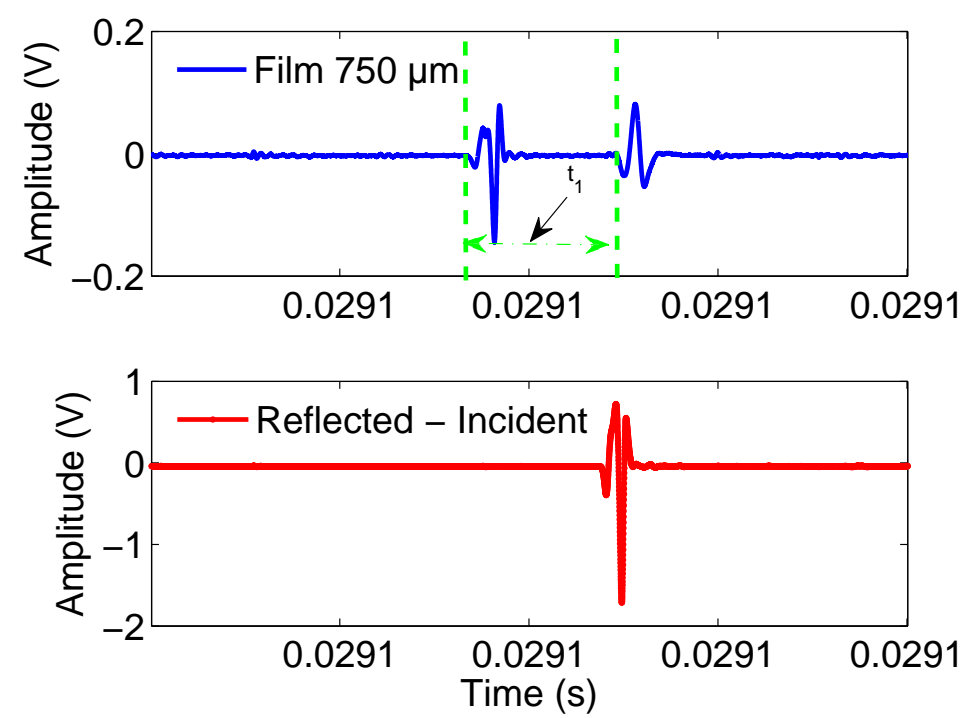

(a)

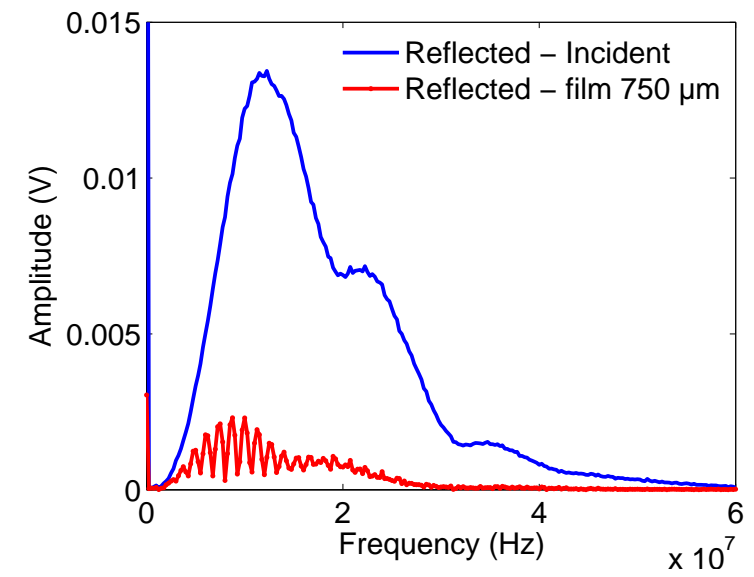

(b)

Figure 7: (a) The reflected temporal signals and (b) their frequency spectra for the $750 \mu \mathrm{m}$ thick PVB film. The time of flight in the thin film layer is denoted $t_{1}$. 


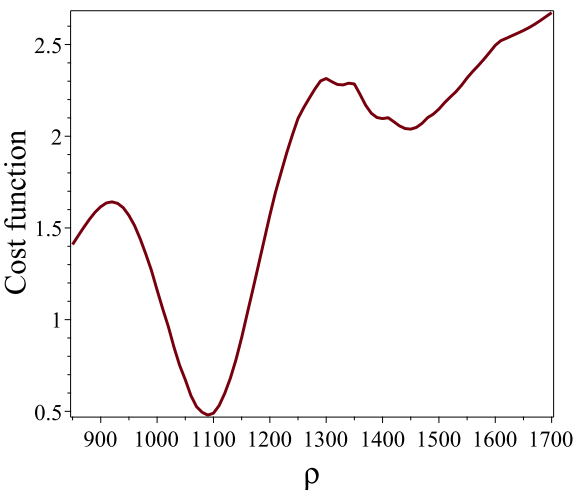

(a)

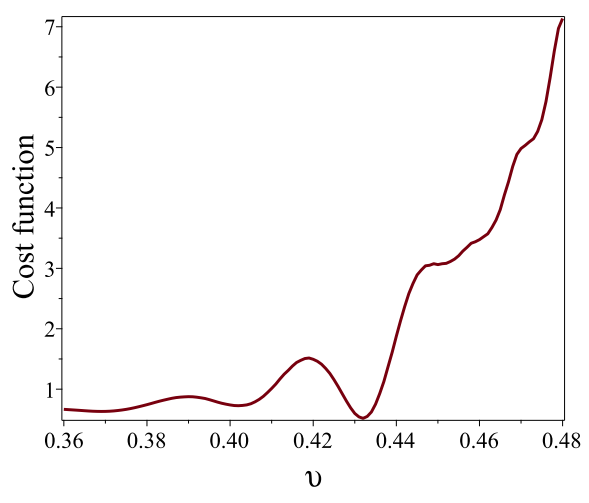

(c)

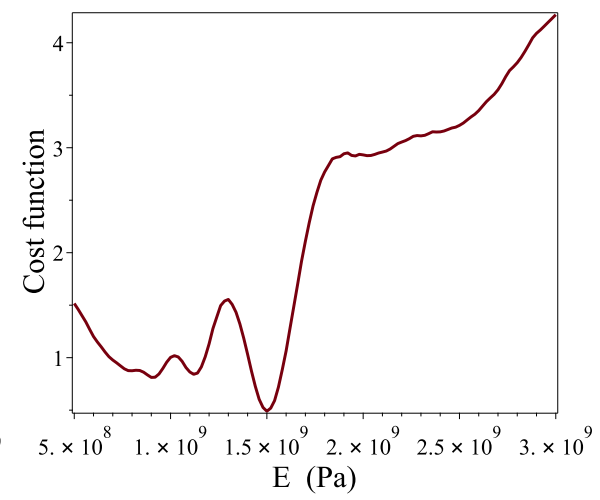

(b)

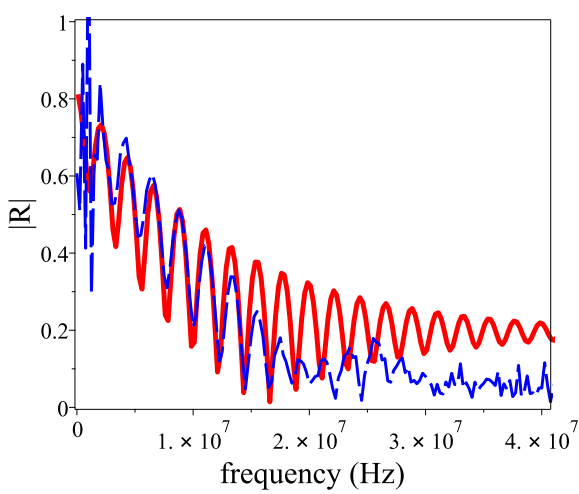

(d)

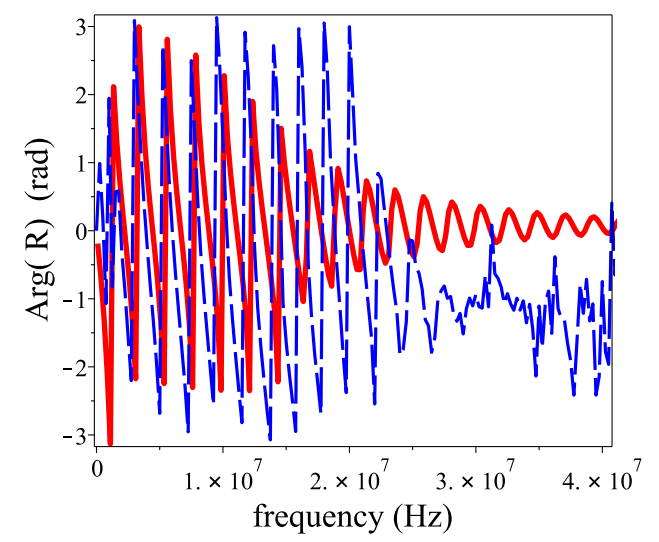

(e)

Figure 8: The cost functions for the recovery of the three mechanical parameters for a $450 \mu \mathrm{m}$ PVB film on a $8 \mathrm{~mm}$ thick glass substrate with sliding contact boundary condition (a) the density $\rho\left(\mathrm{kg} / \mathrm{m}^{3}\right)$, (b) Young's modulus, (c) Poisson ratio. The comparison between the theoretical (continuous line) computed using the recovered parameters and the experimental one (dot-dash line) where in (d) the magnitude and (e) the argument of the reflection coefficient $R$. 


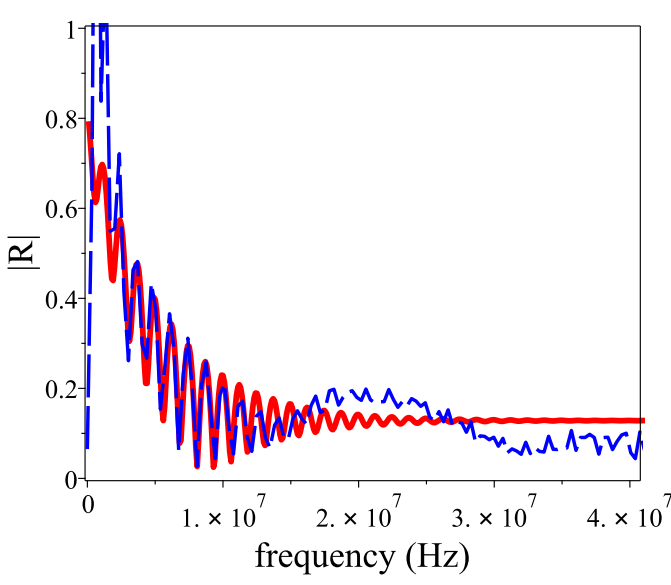

(a)

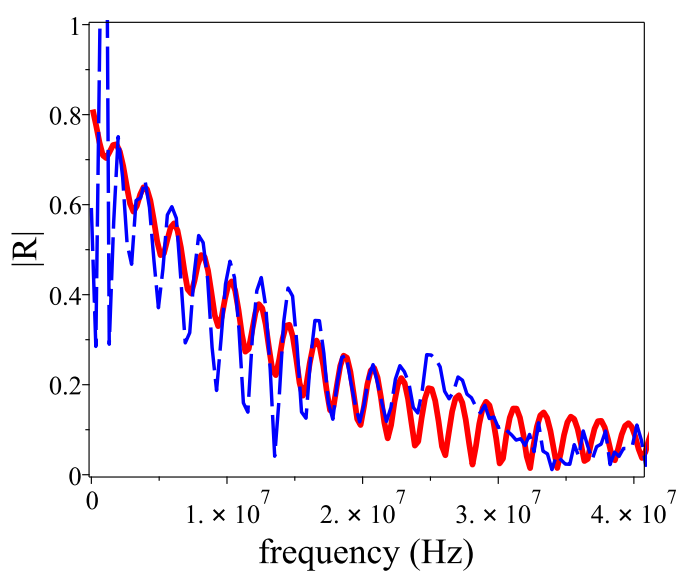

(c)

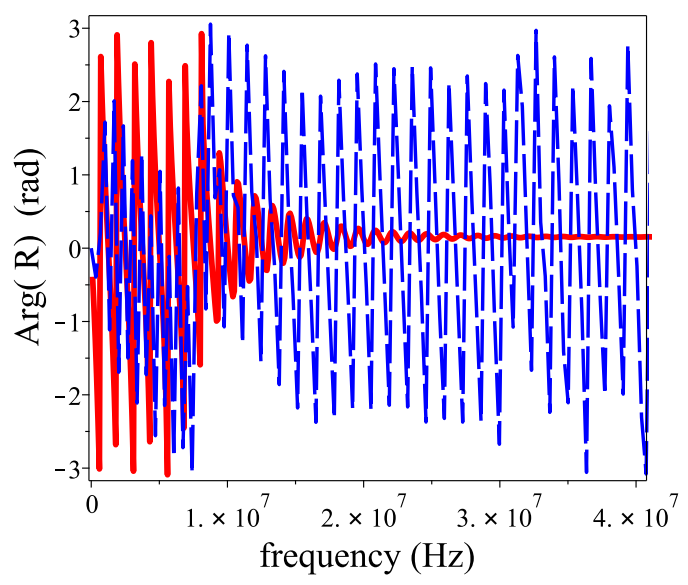

(b)

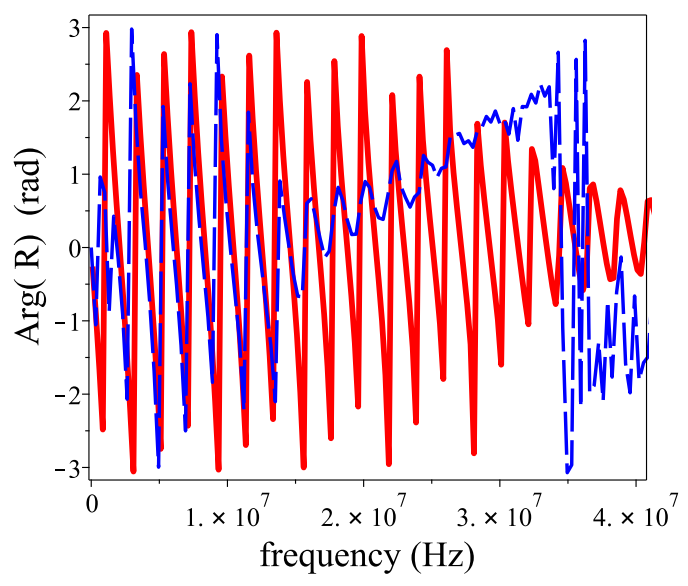

(d)

Figure 9: The comparison between the reflection coefficients obtained using the theoretical interaction model (continuous line) with values of parameters retrieved from the measured data (dot-dash line) for the PVB films placed on a thick glass plate $(8 \mathrm{~mm}$ thick) substrate. The interfaces between the PVB films and the glass were considered as sliding. (a) and (b) are the magnitude and argument respectively of the reflection coefficient for the $750 \mu \mathrm{m}$ thick film, (c) and (d) are those for the $400 \mu \mathrm{m}$ thick PVB film.

\subsection{Summary of the results}

The results are summarized in Table (1). The densities were compared with those obtained from small specimens of the film whose weights were measured on an analytic electronic balance and the volume measured using a cylindrical tube. The velocities measured from the time of flight using correlation functions and the measured densities 


\begin{tabular}{|ccccccccc|}
\hline & Thickness $(\mu \mathrm{m})$ & \multicolumn{2}{c}{ Velocity $(\mathrm{m} / \mathrm{s})$} & $E_{1}(\mathrm{GPa})$ & $\nu_{1}$ & Loss & \multicolumn{2}{c|}{$\rho_{1}\left(\mathrm{~kg} / \mathrm{m}^{3}\right)$} \\
& & measured $\left(v_{L}\right)$ & $\mathrm{IP}\left(v_{11}\right)$ & & & $\eta_{s 1}$ & IP & Measured \\
& 750 & 1895 & 1872 & 1.25 & 0.43 & 0.08 & 1020 & $1086-1101$ \\
PVB & 450 & 1941 & 1996 & 1.5 & 0.432 & 0.06 & 1090 & 1097 \\
& 400 & 1648 & 1668 & 1.12 & 0.42 & 0.05 & 1027 & 1083 \\
\hline PET & 100 & 1851 & 1848 & 2.15 & 0.414 & 0.005 & 1462 & 1497 \\
PCL triol & 200 & 2267 & 2259 & 3.77 & 0.36 & 0.0075 & 1260 & $1278-1420$ \\
\hline
\end{tabular}

Table 1: Summary of the identified mechanical parameters with the measured velocity recovered from the time of flight of the longitudinal wave in the film layer.

results were within $3 \%$ (percent) difference with the ones recovered by solving the inverse problem using experimental reflection coefficient data.

The values of the set of parameters used for computation in the case of the aluminium substrate are $\left(E_{2}=67 \mathrm{GPa}, \nu_{2}=0.3, \rho_{2}=2780 \mathrm{~kg} / \mathrm{m}^{3}\right)$ and those for glass are $\left(E_{2}=\right.$ $\left.70 \mathrm{GPa}, \nu_{2}=0.22, \rho_{2}=2500 \mathrm{~kg} / \mathrm{m}^{3}\right)$.

\subsection{Case of a thin PET film with sliding BC on substrates made of different elastic materials}

The comparison between the modeled reflection coefficient with the measured one for the $100 \mu \mathrm{m}$ PET film resting on three different substrates: a $8 \mathrm{~mm}$ thick glass plate substrate, a rough surface aluminium slab substrate and aluminium cylinder substrate are shown in Fig. (10). The interface is considered as sliding in the theoretical model. The best material substrates that approaches closest the modeled reflection coefficient amplitude is the aluminium one. Young's modulus, Poisson coefficient, density for the $100 \mu \mathrm{m}$ thick film are; $2.15 \mathrm{GPa}, 0.41,1490 \mathrm{~kg} / \mathrm{m}^{3}$ respectively. The similarities of these values to those in Table (11) indicate that the method of posing the film on the substrate without gluing is reliable and can be employed easily to characterize most flexible thin and soft materials. 


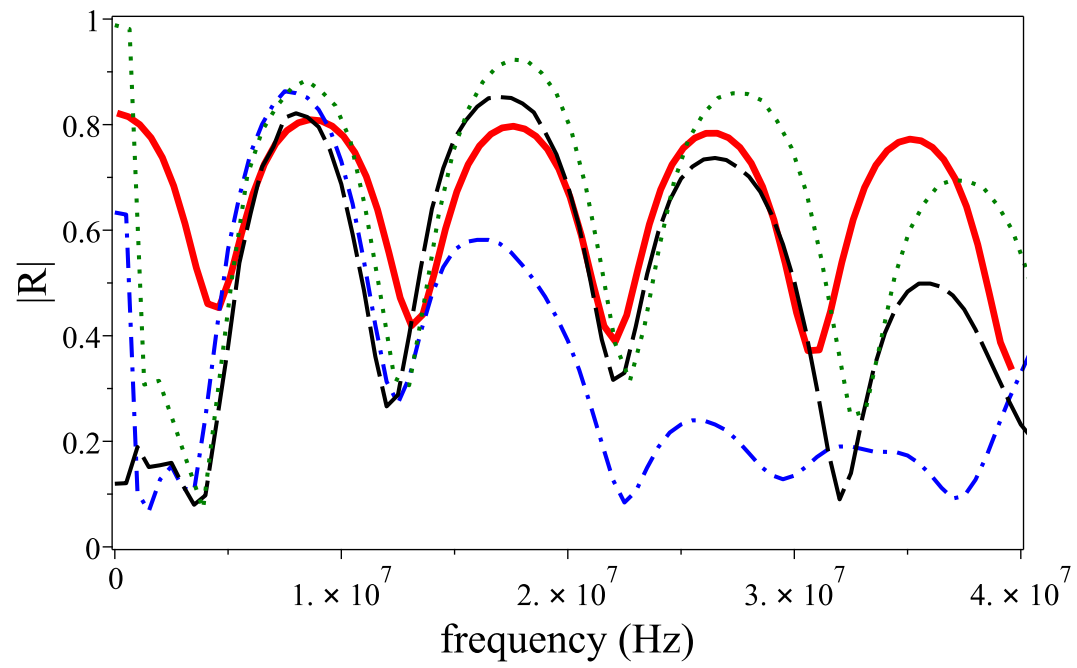

Figure 10: The comparison between the model when the interface is considered as sliding (solid line), the measured reflection coefficients for the $100 \mu \mathrm{m}$ PET film resting on the glass plate substrate (dotted line), aluminium slab substrate (long-dash) and rough surface aluminium block substrate (dash-dot).

\section{Discussion}

The difficulty in making the incident pressure (reflected acoustic wave from the opposite side of the elastic substrate employed as an acoustic mirror) to coincide with the reflected pressure from the thin film is due to the deformation of the latter by the heavier substrate lying on top of it. This deformation of the thin film can avoided or improved if the elastic substrate is chosen in such a way that it is not too heavy. The consequence of the thin film deformation is the increase of the substrate - transducer distance. The other elastic substrates (2cm thick wrought iron plate) that were tested gave very similar results. The best choice of elastic material in this experiment was aluminium. Its density is lower than that of most of the elastic materials tested as compared to the heavier wrought iron with the same geometry.

The method chosen to compute the experimental reflection coefficient by employing windowing functions assures that it is not affected by the difference in transducer-film and transducer-mirror/reflector distances during signal acquisition.

The new method for the characterization of the thin PVB film resting on a substrate 
(slip boundary interface) can also be used to study the bulk elastic modulus of plant leaf tissue [23] which have previously been determined using the volume of symplasmic water and the pressure potential [24]. This modulus expresses the change in turgor of tissue cells for a uniform change in the relative water content of the cells. On the other hand, the methods in the cited papers require more complicated apparatus.

\section{Conclusion}

Two methods for the recovery of the mechanical parameters of flexible thermoplastic thin films (PET, PCL Triol, and PVBs) on elastic substrates using high frequency, reflected ultrasonic acoustic waves and the corresponding interaction models using elasticity theory, have been developed. In the first method, the thin film was stuck/bonded onto an elastic substrate using an acrylic glue. The thickness of the glue was neglected. In the second method, the thin flexible film was just placed to rest on the substrate. The interface condition for the first configuration was modeled using bonded boundary condition and in the second case, the thin-films (PVBs and a PET) were placed in contact with the elastic substrate. Subsequently the interface was modeled using contact slip boundary condition. The ultrasonic wave was generated using a $50 \mathrm{MHz}$ transducer. The whole test rig was placed in a water tank. The same transducer was used to excite and acquire the reflected ultrasonic waves from the thin films on their substrates. The method of acquiring the incident signal and the shifting corrections applied to obtain the correct reflection coefficients and wave velocity automatically within the thin films were also developed. Data from the two configurations were employed successfully with the interaction models of the thin films on their substrates to retrieve their mechanical parameters. The retrieved mechanical parameters results agree well with those reported in the literature for materials of the same composition. 


\section{Acknowledgments}

The authors would like to thank Gwendoline Ogam for doing the illustration art. The Algerian government for the grant that supported H. Lazri during his stay at LMA, Marseilles France. B. Mourot of LMA for helping with the administrative paper work. The Centre de ressources Ultrasons of the LMA for the provision of the water tank employed for conducting the measurements. 


\section{AppendixA. The resulting matrix equations}

The relationships employed for the displacement $(u)$, velocity $(v)$ using the wave potentials in the Laplace domain $(s)$ are

$$
\begin{aligned}
u_{x}^{s n} & =\frac{\partial}{\partial x} \Psi^{s n}(x, z, s)-\frac{\partial}{\partial z} \Phi^{s n}(x, z, s), n=1,2, \\
v_{x}^{s n}= & s \frac{\partial}{\partial x} \Psi^{s n}(x, z, s)-s \frac{\partial}{\partial z} \Phi^{s n}(x, z, s), \\
u_{z}^{s n} & =\frac{\partial}{\partial z} \Psi^{s n}(x, z, s)+\frac{\partial}{\partial x} \Phi^{s n}(x, z, s), \\
v_{z}^{s n}= & s \frac{\partial}{\partial z} \Psi^{s n}(x, z, s)+s \frac{\partial}{\partial x} \Phi^{s n}(x, z, s) .
\end{aligned}
$$

The stresses are given by

$$
\begin{gathered}
\sigma_{z z}^{s n}=\lambda_{n}\left(\frac{\partial}{\partial x} u_{x}^{s n}(x, z, s)+\frac{\partial}{\partial z} u_{z}^{s n}(x, z, s)\right)+2 \mu_{n} \frac{\partial}{\partial z} u_{z}^{s n}(x, z, s), n=1,2 \\
\sigma_{x z}^{s n}=\mu_{n}\left(\frac{\partial}{\partial z} u_{x}^{s n}(x, z, s)+\frac{\partial}{\partial x} u_{z}^{s n}(x, z, s)\right) .
\end{gathered}
$$

AppendixA.1. The thin film glued onto the substrate, the matrix equation

The matrix equation is written as a linear system in the form $\mathbf{A} \cdot \mathbf{x}=\mathbf{b}$. The matrix expressions are given in Eqn. A.3. 


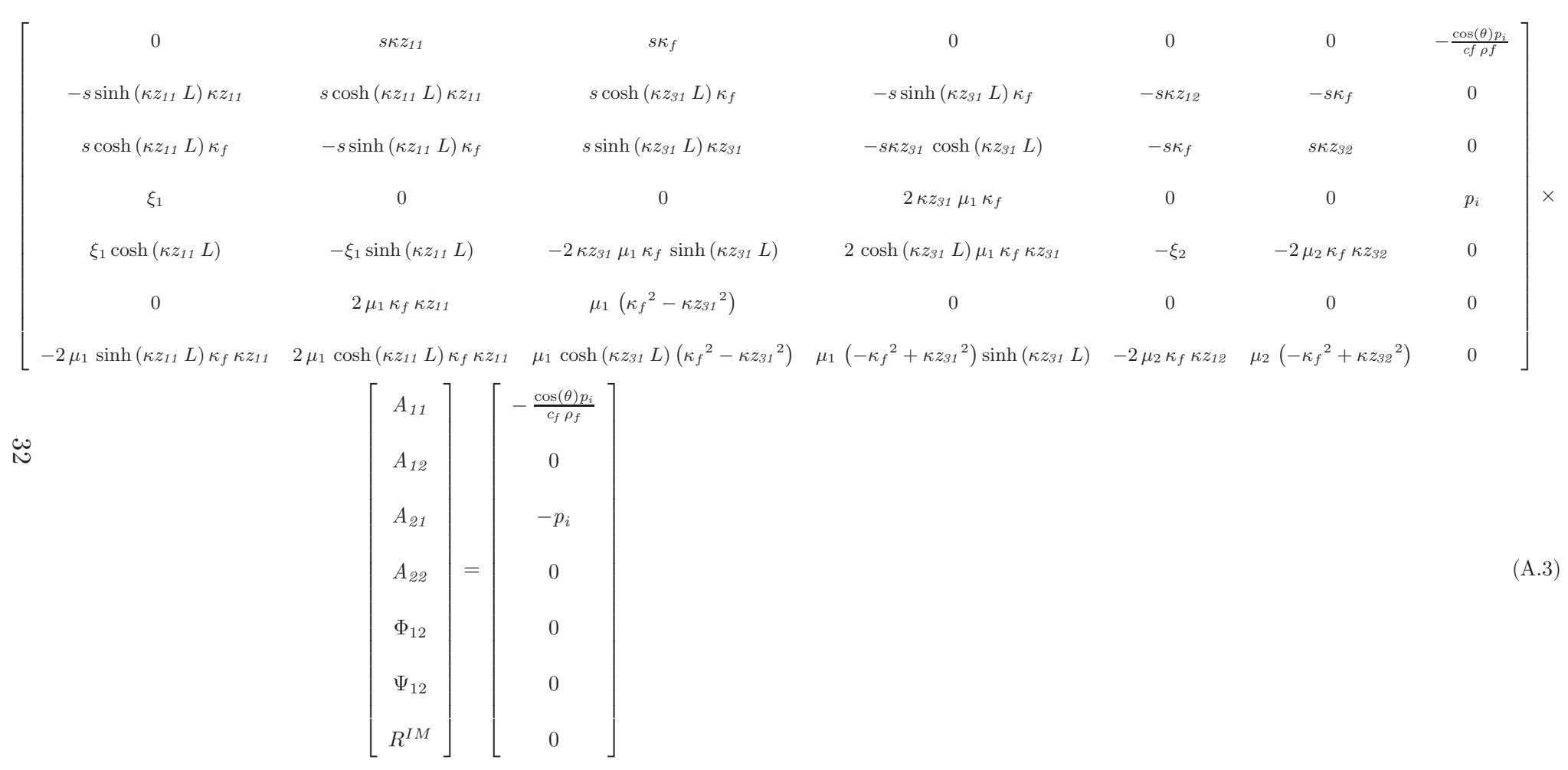


where $\xi_{1}=\left(\left(2 \mu_{1}+\lambda_{1}\right) \kappa z_{11}^{2}+\lambda_{1} \kappa_{f}^{2}\right)$ and $\xi_{2}=\left(2 \mu_{2}+\lambda_{2}\right) \kappa z_{12}{ }^{2}+\lambda_{2} \kappa_{f}^{2}$.

AppendixA.2. The sliding interface case - the matrix equation

The matrix equation for the slip boundary condition case is reported in Eqn.(A.4). 


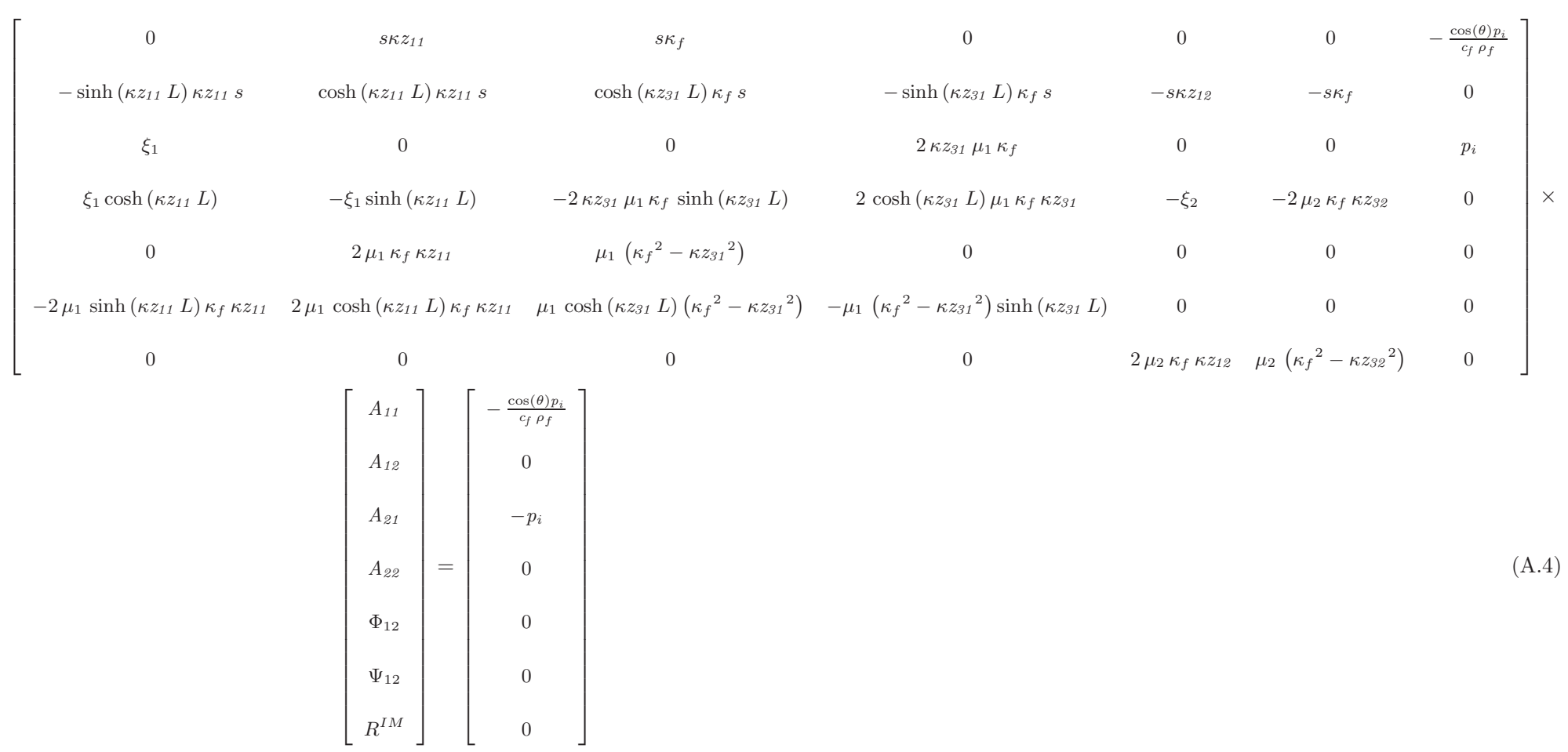


[1] F.-A. E. Toufaili, Catalytic and mechanistic studies of polyethylene terephthalate synthesis, Ph.D. thesis, Technische Universität Berlin (2006).

URL https://doi.org/10.14279/depositonce-1333

[2] X. Zhang, H. Hao, Y. Shi, J. Cui, The mechanical properties of polyvinyl butyral (pvb) at high strain rates, Construction and Building Materials 93 (2015) $404-415$.

URL http://www.sciencedirect.com/science/article/pii/S0950061815006649

[3] V. Chapuis, S. Pélisset, M. Raeis-Barnéoud, H.-Y. Li, C. Ballif, L.-E. Perret-Aebi, Compressiveshear adhesion characterization of polyvinyl-butyral and ethylene-vinyl acetate at different curing times before and after exposure to damp-heat conditions, Progress in Photovoltaics: Research and Applications 22 (4) (2014) 405-414.

URL http://dx.doi.org/10.1002/pip.2270

[4] D. W. v. Krevelen, K. t. Nijenhuis, Properties of Polymers : Their Correlation with Chemical Structure; Their Numerical Estimation and Prediction From Additive Group Contributions., Vol. 4th, completely rev. ed. revised by K. te Nijenhuis, Elsevier Science, 2009.

[5] S. Suresh, T. C. Lim, J. Kastner, Predicting acoustic transmission loss through laminated glass with air and porous layers, International journal of vehicle noise and vibration 8 (3) (2012) 237-260.

[6] T. Deichmann, M. G. McCord, M. A. Bourham, T. Gries, Analysis of atmospheric pressure plasma parameters during treatment of polyethylene terephthalate films, Journal of Applied Polymer Science 121 (4) (2011) 1875-1884.

URL http://dx.doi.org/10.1002/app.33557

[7] A. Briggs, O. Kolosov, Acoustic Microscopy, Oxford University Press, 2009. URL http://dx.doi.org/10.1093/acprof : oso/9780199232734.001.0001

[8] M. Maebayashi, T. Matsuoka, S. Koda, R. Hashitani, T. Nishio, S. ichi Kimura, Study on polystyrene thin film on glass substrate by scanning acoustic microscope, Polymer 45 (22) (2004) $7563-7569$.

URL http://www.sciencedirect.com/science/article/pii/S0032386104008705

[9] A. I. Lavrentyev, S. I. Rokhlin, An ultrasonic method for determination of elastic moduli, density, attenuation and thickness of a polymer coating on a stiff plate, Ultrasonics 39 (3) (2001) $211-221$. URL http://www.sciencedirect.com/science/article/pii/S0041624X00000664

[10] J. Lim, M. Ratnam, I. Azid, D. Mutharasu, Determination of Young's modulus of epoxy coated polyethylene micro-cantilever using phase-shift shadow Moiré method, Optics and Lasers in Engineering 49 (11) (2011) $1301-1308$.

URL http://www.sciencedirect.com/science/article/pii/S0143816611001710

[11] E. Ogam, Z. Fellah, G. Ogam, Identification of the mechanical moduli of closed-cell porous foams using transmitted acoustic waves in air and the transfer matrix method, Composite Structures 135 
(2016) $205-216$.

URL http://www.sciencedirect.com/science/article/pii/S026382231500865X

[12] L. M. Brekhovskikh, Waves in layered Media, Academic Press, New York, NY, USA, 1960.

[13] J.-P. Groby, E. Ogam, L. D. Ryck, N. Sebaa, W. Lauriks, Analytical method for the ultrasonic characterization of homogeneous rigid porous materials from transmitted and reflected coefficients, J. Acoust. Soc. Am. 127 (2) (2010) 764-772.

[14] MapleSoft, Maple 16 User Manual, Maplesoft, a division of Waterloo Maple Inc., Ontario, Canada (2016).

[15] M. Ben Mansour, E. Ogam, Z. E. A. Fellah, A. Soukaina Cherif, A. Jelidi, S. Ben Jabrallah, Characterization of compressed earth blocks using low frequency guided acoustic waves, J. Acoust. Soc. Am. 139 (5) (2016) 2551-2560. URL http://scitation.aip.org/content/asa/journal/jasa/139/5/10.1121/1.4948573

[16] T.-C. Li, B.-H. Wu, J.-F. Lin, Effects of pre-strain applied at a polyethylene terephthalate substrate before the coating of tio2 film on the coating film quality and optical performance, Thin Solid Films 519 (22) (2011) $7875-7882$.

URL http://www.sciencedirect.com/science/article/pii/S0040609011005682

[17] L. Bistričić, V. Borjanović, M. Leskovac, L. Mikac, G. E. McGuire, O. Shenderova, N. Nunn, Raman spectra, thermal and mechanical properties of poly(ethylene terephthalate) carbon-based nanocomposite films, Journal of Polymer Research 22 (3) (2015) 39.

URL http://dx.doi.org/10.1007/s10965-015-0680-z

[18] G.-I. Shim, S.-H. Kim, D.-L. Ahn, J.-K. Park, D.-H. Jin, D.-T. Chung, S.-Y. Choi, Experimental and numerical evaluation of transparent bulletproof material for enhanced impact-energy absorption using strengthened-glass/polymer composite, Composites Part B: Engineering 97 (2016) 150 - 161. URL http: //www.sciencedirect.com/science/article/pii/S1359836816305571

[19] E. Ogam, Z. Fellah, N. Sebaa, J.-P. Groby, Non-ambiguous recovery of Biot poroelastic parameters of cellular panels using ultrasonic waves, J. Sound Vibr. 330 (6) (2011) 1074 - 1090.

[20] W. Liang, L. Chen, F.-x. Zhou, Z.-H. Ge, G. Ding, Maximum fraction cross-correlation spectrum for time of arrival estimation of ultrasonic echoes, Russian Journal of Nondestructive Testing 51 (2) (2015) 120-130.

[21] I. MathWorks, Signal Processing Toolbox for Use with MATLAB: User's Guide, version 7.2 (r2016a) Edition, Computation, visualization, programming, The MathWorks, Natick, MA USA, 2016.

[22] Wikipedia, Window function — wikipedia, the free encyclopedia, [Online; accessed 2-May-2017] (2017).

URL https://en.wikipedia.org/w/index.php?title=Window_function\&oldid=778173249

[23] R. López, J. Rodríguez-Calcerrada, L. Gil, Physiological and morphological response to water deficit 
in seedlings of five provenances of pinus canariensis: potential to detect variation in droughttolerance, Trees 23 (3) (2009) 509-519.

URL http://dx.doi.org/10.1007/s00468-008-0297-5

[24] T. Saito, K. Soga, T. Hoson, I. Terashima, The bulk elastic modulus and the reversible properties of cell walls in developing quercus leaves, Plant and Cell Physiology 47 (6) (2006) 715-725.

URL http://pcp.oxfordjournals.org/content/47/6/715.abstract 